\title{
Correcting the Variations of BOLD Signal Due to Susceptibility Gradients and Its Application
}

\author{
Lifang Zhao1, Lianjun Zhang², Gang Liu ${ }^{2}$ \\ ${ }^{1}$ Library, Shandong University of Technology, Zibo, China \\ ${ }^{2}$ School of Computer, Shandong University of Technology, Zibo, China \\ Email: zhlj0512@163.com
}

How to cite this paper: Zhao, L.F., Zhang, L.J. and Liu, G. (2019) Correcting the Variations of BOLD Signal Due to Susceptibility Gradients and Its Application. Journal of Computer and Communications, 7, 20-32. https://doi.org/10.4236/jcc.2019.711002

Received: September 20, 2019

Accepted: November 2, 2019

Published: November 5, 2019

Copyright $\odot 2019$ by author(s) and Scientific Research Publishing Inc. This work is licensed under the Creative Commons Attribution International License (CC BY 4.0).

http://creativecommons.org/licenses/by/4.0/

(c) (i) Open Access

\begin{abstract}
Blood oxygenation level dependence signal (BOLD) for functional magnetic resonance imaging (FMRI), is the use of blood magnetization depending on the oxygenation state of hemoglobin. Susceptibility gradient can shift and skew $k$-space trajectories and it leads to echo time shift and BOLD sensitivity change. FMRI can be used to detect the signal, the change of the susceptibility gradient of the signal and the distortion of $\mathrm{k}$ space trajectory, resulting in echo time shift and BOLD sensitivity change. Using the percentage signal change (PSC) and calibration function, it can be applied to many different fields, such as age-related research. In this paper, the performance of BOLD signal change correction based on sensitivity gradient was verified by real data group calculation, and methods of further improving the calculation speed were analyzed. This paper also analyzed the performance of correcting the variations of BOLD Signal due to susceptibility gradients with real data set, and identified the computational issues that need to be improved for further research.
\end{abstract}

\section{Keywords}

BOLD Signal, Susceptibility Gradients, FMRI, Percentage Signal Change

\section{Introduction}

Functional magnetic resonance imaging (FMRI), using blood oxygenation level dependent signal (BOLD), is the most pervasive tool in cognitive neuroscience to non-invasively examine which parts of the brain are involved in which functions and to look at changes in function across the lifespan. This is done by relying on the magnetic susceptibility of blood that depends on the oxygenation state of hemoglobin. As neuronal activity provokes an increase in oxygen consump- 
tion and blood flow, the number of oxygenated hemoglobin will increase and the number of deoxygenated hemoglobin will decrease in that region which will lead to a paramagnetic effect and MRI can detect this $T_{2}^{*}$-weighted signal.

Since FMRI relies on the magnetic susceptibility, it is sensitive to the microscopic magnetic susceptibility variations in blood. However, FMRI is also sensitive to the macroscopic magnetic susceptibility differences from air/tissue interfaces which cause artifacts. A large magnetic susceptibility difference existing between air and tissue leads to severe disruptions of the uniformity of the magnetic field around that area. The susceptibility-induced magnetic field inhomogeneity causes image distortion and signal loss. There have been many developments and optimizations to correct those artifacts from susceptibility magnetic field inhomogeneity [1] [2] [3] [4].

An additional artifact that has received much less examination is the effect of gradients in the magnetic field on the sensitivity of the BOLD FMRI acquisition [1]. Gradients in the magnetic field due to macroscopic field inhomogeneity can disrupt the spatial-frequency encoding of a voxel, resulting in a shift in the effective echo time for that voxel. This effect results in each voxel in a functional image having a potentially different effective echo time. Since the BOLD signal is a function of the echo time of the acquisition, this can result in a spatially varying BOLD signal even if the underlying activity was uniform. It is this artifact that is the focus of the current manuscript.

This spatially-varying BOLD sensitivity potentially depends on: the protocol (acquisition trajectory, acquisition timing, slice orientations, phase encode direction), the subject (specific anatomy of air/tissue interfaces or iron deposition), and the experimental details (quality of the shimming and orientation of the subjects head in the scanner). In an aging study, differences between young and old subjects could exist in the anatomy and orientation of air/tissue interfaces along with changes in magnetic field maps due to iron concentrations.

In this research, we are going to calibrate changes in BOLD sensitivity that are due to changes in the magnetic field and delineate regions that show significant sensitivity changes with age. By comparing the BOLD sensitivity between young and old subjects, we determine some areas in a brain that have significant differences in functional sensitivity related with age.

\section{Methods and Data Analysis}

\subsection{Magnetic Resonance Imaging}

Tomography is a tool that we can use to create an internal image of the body without cutting it open. There have been many different techniques to create internal images and one of the active ongoing areas is magnetic resonance imaging (MRI). MRI is based on the physics of nuclear magnetic resonance (NMR) which is a phenomenon that nuclei having an odd number of neutrons, protons, or both have a magnetic moment. If there is no magnetic field, then all the magnetic moments will spin in random directions and the sum of them will be zero. 
However, if there is a static magnetic field, $\boldsymbol{B}_{0}$, then the magnetic moments will have a net alignment and a net magnetization, $\boldsymbol{M}$, will be aligned either parallel or anti-parallel to the magnetic field. When the net magnetization is tipped out of alignment by an RF pulse, it precesses around the magnetic field with the Larmor frequency:

$$
w_{0}=\gamma B_{0}
$$

here $B_{0}$ is the strength of the magnetic field and $\gamma$ is the gyromagnetic ratio. Generally, $\gamma=\frac{\gamma}{2 \pi}$ is widely used. For example, with $1 \mathrm{H}$ in the magnetic field, $\gamma=42.58 \mathrm{MHz} / \mathrm{T}$.

If a radio frequency $(\mathrm{RF})$ pulse is applied, the spins that have the same frequency as the RF pulse will absorb energy and become excited. An RF pulse is a $B_{1}$ field that oscillates perpendicular to the $B_{0}$ field. The consequence of excitation is the tip-down of the net magnetization. The flip angle, $\alpha$, is determined by the strength, $B_{1}$, and the duration, $\tau_{p}$, of RF pulse.

$$
\alpha=\int_{0}^{\tau_{p}} \gamma B_{1}(t) \mathrm{d} t
$$

After the short pulse, the net magnetization will be resurned to the equilibrium which is called relaxation. The longitudinal magnetization recovery is called longitudinal relaxation and the transverse magnetization decay is called transverse relaxation. The recovery rate of the longitudinal magnetization is characterized by the tissue-specific time constant $T_{1}$. After time $T_{1}$, longitudinal magnetization has returned to $63 \%$ of its final value. The decay rate of the transverse magnetization is characterized by the time constant $T_{2}$. After time $T_{2}$, transverse magnetization has lost $63 \%$ of its original value. $T_{2}$ is tissuespecific and is always shorter than $T_{1}$ because transverse relaxation is faster than longitudinal relaxation.

$$
\begin{gathered}
M_{x y}(t)=M_{x y}\left(0_{+}\right) \mathrm{e}^{-t / T_{2}} \mathrm{e}^{-i w_{0} t} \\
M_{z}(t)=M_{z}^{0}\left(1-\mathrm{e}^{-t / T_{1}}\right)+M_{z}\left(0_{+}\right) \mathrm{e}^{-t / T_{1}}
\end{gathered}
$$

Free induction decays (FID) are the signals recorded without magnetic gradients. If the magnetic field is inhomogeneous, then FID signals will decay faster because magnetic moments can cancel out each other with a small magnetic field differences and the decay of magnetization is given a time constant called $T_{2}^{*}$ which is smaller than $T_{2}$.

\subsection{Functional MRI}

Functional MRI (FMRI) detects the signal changes due to neural activities in a brain. Deoxygenated hemoglobin is paramagnetic and fully oxygenated hemoglobin is diamagnetic, which is more similar to the surrounding tissue. The deoxygenated hemoglobin during neural activities distorts the static magnetic field more. The MRI signal will decay because the different frequencies that process in non-uniform magnetic field cause rapid phase cancellation. Therefore, changes 
in blood oxygenation cause changes in the MR decay parameter, $T_{2}^{*}$, and it also changes the $T_{2}^{*}$-weighted image. This technique is called blood oxygenation level dependent (BOLD) contrast.

FMRI is performed by acquiring a time series of snapshot images of the brain, typically every two seconds for several minutes. During this time, subjects are presented with visual-based or other stimuli that change throughout the scan. Examining how the measured brain signal correlates with the presented stimuli will reveal which areas of the brain are undergoing activity, and associated changes in the concentration of deoxygenated hemoglobin.

\subsection{Sampling Trajectories for Functional MRI}

In order to acquire the necessary samples to satisfy Nyquist sampling in the data space, or $k$-space, several acquisition trajectories are used. Trajectories are acquired by playing out gradients that travel through the spatial-frequency space of $k$-space. Location in $k$-space is given by integrating the gradients,

$$
\boldsymbol{k}(t)=\gamma \int_{0}^{t} \boldsymbol{G}(\tau) \mathrm{d} \tau
$$

Signals are recorded in $k$-space, $S(\boldsymbol{k})$, which is the Fourier transform of a spatial function, $\rho(\boldsymbol{r})$.

$$
S(\boldsymbol{k})=\mathcal{F}\{\rho(\boldsymbol{r})\}=\int_{-\infty}^{\infty} \cdots \int_{-\infty}^{\infty} \rho(\boldsymbol{r}) \mathrm{e}^{-i 2 \pi k \cdot r} \mathrm{~d} \boldsymbol{r}
$$

If we want to reconstruct the spatial function which is the original image, we can perform inverse Fourier transform of $k$-space.

$$
\rho(\boldsymbol{r})=\int_{-\infty}^{\infty} \cdots \int_{-\infty}^{\infty} S(\boldsymbol{k}) \mathrm{e}^{i 2 \pi \boldsymbol{k} \cdot \boldsymbol{r}} \mathrm{d} \boldsymbol{k}
$$

There are several ways that you can traverse $k$-space with different trajectories. One method is echo-planar imaging (EPI) trajectory, as shown in Figure 1. The trajectory starts from a corner of $k$-space and it only moves to $y$-direction once it reaches the border of $k$-space on $x$-direction. According to the sampling theorem,

$$
\Delta k_{x} \leq \frac{1}{F O V_{x}}, \Delta k_{y} \leq \frac{1}{F O V_{y}}
$$

where $F O V_{x}$ is the field of view in the $x$-direction and $\Delta k_{x}$ is the sample spacing in the $x$-direction of $k$-space. If this sampling is not satisfied, there will be aliasing in the image. EPI trajectory can cover all $k$-space and sample uniformly. However, this is not the time-efficient method, because slew rate reduces the sampling rate when the $y$-value is changed and the direction on the $x$-axis is reversed to cover the next line. There are two ways that EPI can be performed. EPI-down, also called phase-encode anterior to posterior (PEAP), starts the trajectory from the top of the $k$-space to the bottom. EPI-up, also called phase-encode posterior to anterior (PEPA), starts the trajectory from the bottom of the $k$-space to the top. This issue can be raised up if there is susceptibility gradient inhomogeneity and distorts the trajectory which will be covered later in the paper. 


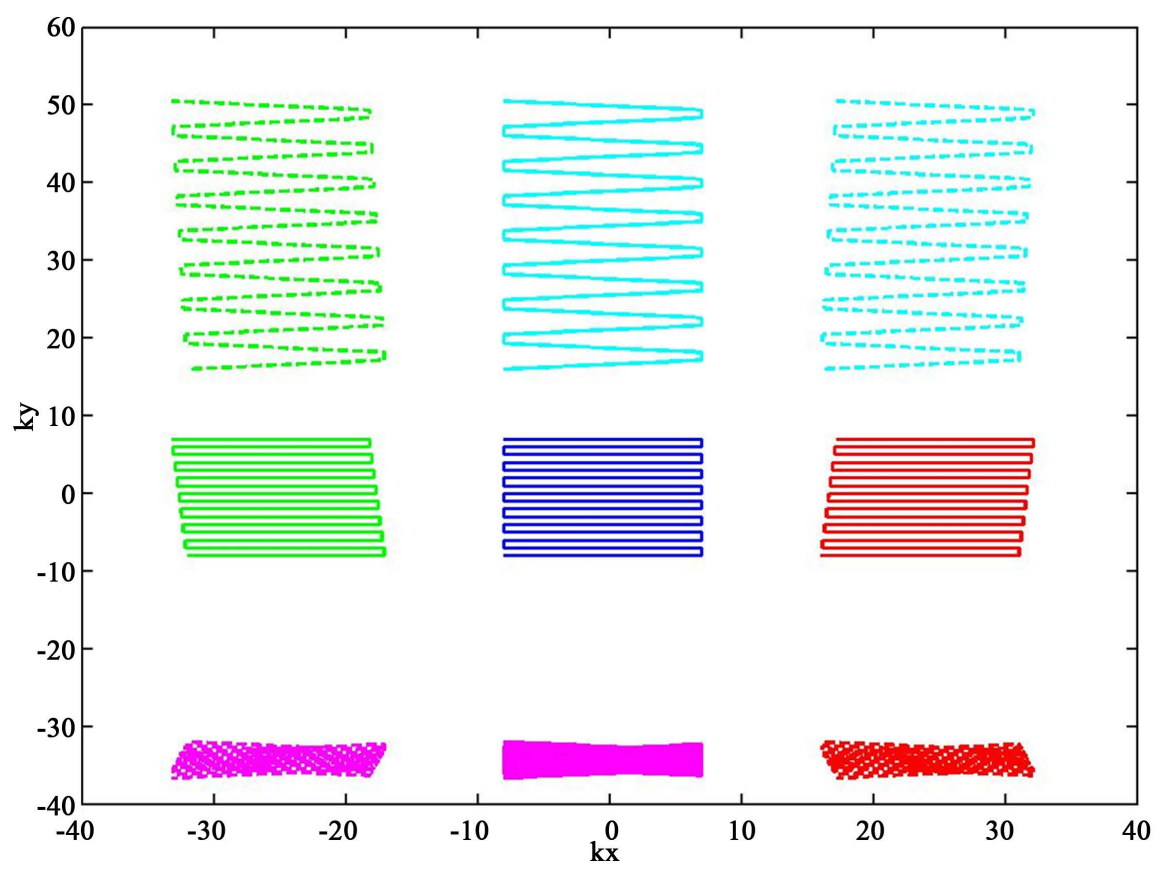

Figure 1. EPI trajectory with susceptibility gradients.

Another $k$-space sampling trajectory is the spiral trajectory, as shown in Figure 2. Liang et al. defined a spiral trajectory in the $\left(k_{x}, k_{y}\right)$-plane as

$$
\boldsymbol{k}(t)=A \omega(t) \mathrm{e}^{i \omega(t)}
$$

where $\boldsymbol{k} \equiv k_{x}+i k_{y}$, and $\omega(t)$ are function of time. A spiral trajectory starts from the origin and draws the spiral toward outside. This trajectory acquires the data faster than EPI trajectory because spiral trajectory does not need to completely change the direction and it does not need to slow down because of slew rate. However, the spiral acquisition shown in Figure 3 does not cover the four corners of the $k$-space in spiral trajectory and it might cause a blur in the image when the spatial function is reconstructed. By the sampling theorem,

$$
\Delta k_{\text {rad }} \leq \frac{1}{F O V_{\text {rad }}}
$$

where $F O V_{\text {rad }}$ is the size of the circular field of view in the radial-direction.

\subsection{Susceptibility}

Before defining magnetic susceptibility, let us define permeability. The permeability, $\mu$, is defined by the relationship between the physical magnetic field, $\boldsymbol{B}$, and another vector field, $\boldsymbol{H}$.

$$
\boldsymbol{B}=\mu \boldsymbol{H}
$$

A relative permeability is defined by $\mu_{r}=\mu / \mu_{0}$ where $\mu_{0}=4 \pi \times 10^{-7}$ is a permeability in empty space. After counting both free current and the magnetization current, $H$ field can be redefined as

$$
\boldsymbol{H}=\boldsymbol{B} / \mu_{0}-\boldsymbol{M}
$$




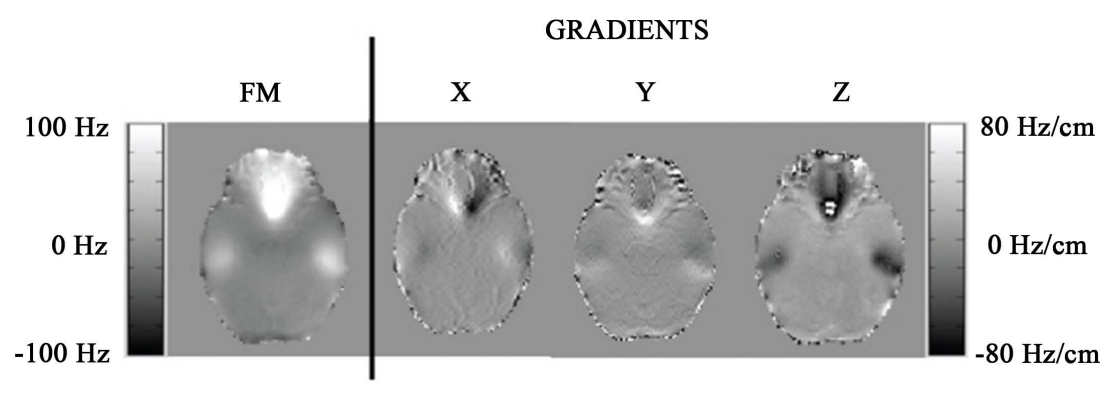

Figure 2. Field map and its gradients in $x, y$, and $z$-direction. The variations in the magnetic field are near brain and air interfaces due to susceptibility differences.

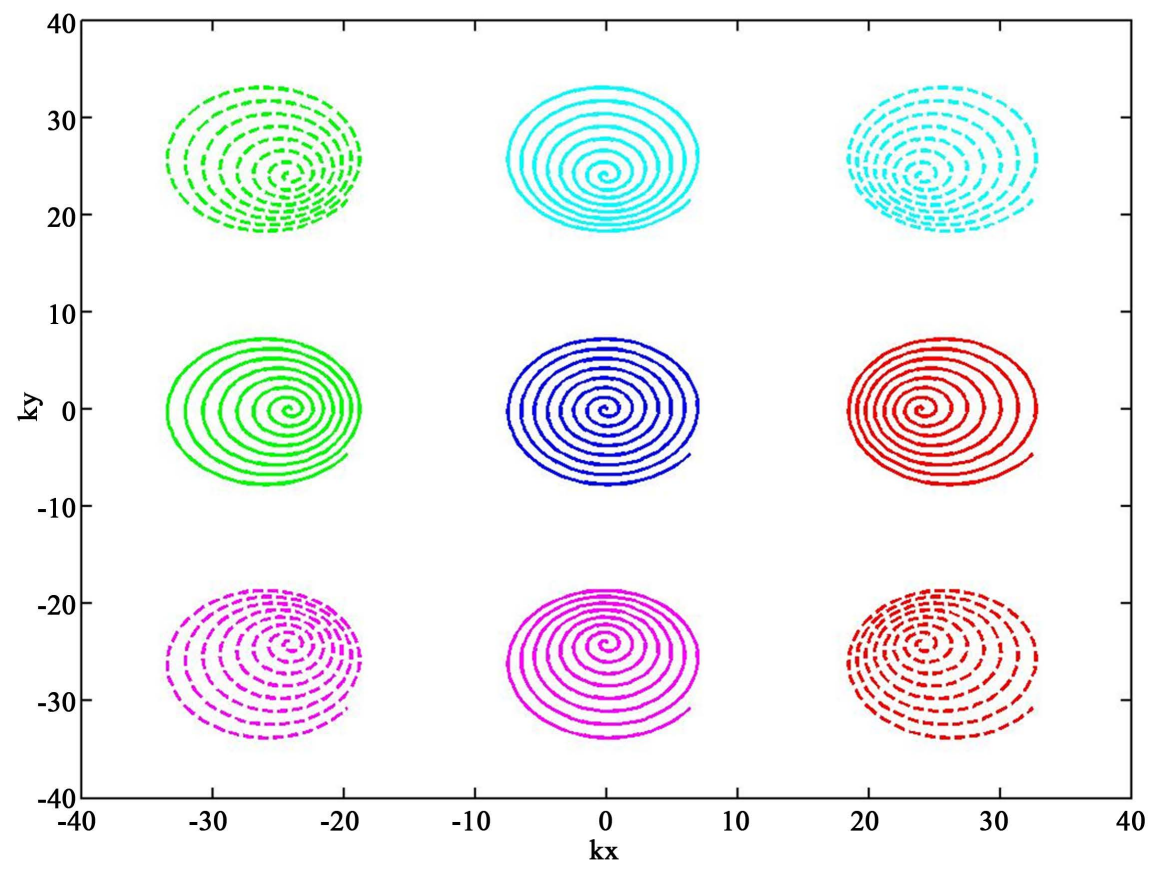

Figure 3. Spiral trajectory with susceptibility gradients.

The magnetic susceptibility, $\chi$, is defined by the relationship between the magnetization, $\boldsymbol{M}$, and $H$ field.

$$
\boldsymbol{M}=\chi \boldsymbol{H}
$$

Therefore, $\chi=\frac{\mu}{\mu_{0}}-1$ and the relationship between $B$ field and $H$ field as

$$
\boldsymbol{B}=(1+\chi) \mu_{0} \boldsymbol{H}
$$

And the relationship between $B$ field and $M$ field is

$$
\boldsymbol{B}=\frac{1+\chi}{\chi} \mu_{0} \boldsymbol{M}
$$

Magnetic susceptibility is a property of specific materials or tissues and can vary between different tissues. This results in a variation of the magnetic field at interfaces between soft tissues $\left(\chi_{\text {tissue }}=-9 \times 10^{-6}\right)$ and air $\left(\chi_{\text {air }}=0.4 \times 10^{-6}\right)$.

Variations of the magnetic field cause image artifacts such as geometric dis- 
tortion and phase effects. The phase variation due to the magnetic field variation at location, $\boldsymbol{r}$, in a given echo time, $T_{E}$, can be expressed as

$$
\phi\left(\boldsymbol{r}, T_{E}\right)=\phi_{0}-\gamma \Delta B(\boldsymbol{r}) T_{E}
$$

where $\phi_{0}$ is a constant phase offset independent of time and $\Delta B(\boldsymbol{r})$ is the presence of local variations in the magnetic field. By scanning with different echo times, $T_{E_{1}}$ and $T_{E_{2}}$, we can extract

$$
\Delta B(\boldsymbol{r})=\frac{\phi\left(\boldsymbol{r}, T_{E_{2}}\right)-\phi\left(\boldsymbol{r}, T_{E_{1}}\right)}{\gamma\left(T_{E_{1}}-T_{E_{2}}\right)}
$$

If we find $\Delta B(\boldsymbol{r})$, then we can also find $\Delta \chi$ between the two tissues.

Figure 2 shows the field map and the corresponding susceptibility gradients in $x, y$, and $z$-directions. The variations in the magnetic field exist at regions in the brain near air/tissue interfaces due to the susceptibility differences of air and tissue. The $k$-space generated by imaging gradients without susceptibility is

$$
K_{\text {imaging }}(t)=\gamma \int_{0}^{t} G_{\text {imaging }}(\tau) \mathrm{d} \tau
$$

After counting the susceptibility gradients, the $k$-space trajectory will be shifted and skewed because the location of $k$-space depends on the susceptibility gradient. The $k$-space generated by susceptibility gradients is

$$
K_{\text {susc }}(t)=\gamma \int_{0}^{t} G_{\text {susc }}(\tau) \mathrm{d} \tau
$$

Since $G_{\text {susc }}$ is a constant $\left(G_{\text {susc }}(t)=G_{\text {susc }} \quad \forall t\right)$, we can simplify the previous formula to

$$
K_{\text {susc }}(t)=\gamma G_{\text {susc }} t
$$

Therefore, $k$-space after counting the susceptibility gradients would be

$$
K(t)=K_{\text {imaging }}(t)+K_{\text {susc }}(t)=\gamma \int_{0}^{t} G_{\text {imaging }}(\tau) \mathrm{d} \tau+\gamma G_{\text {susc }}\left(t+t_{0}\right)
$$

where $t=0$ when the data acquisition starts and $t_{0}$ is the duration between the RF pulse and the beginning of the data acquisition. For example, in EPIdown, which is also called EPI PEAP, the $k$-space trajectory will be shifted and skewed. If the susceptibility gradient is positive which is the same direction as the gradients without susceptibility, the starting point of $k$-space trajectory will be shifted downward because of susceptibility gradients between the RF pulse and the data acquisition. Also, since the trajectory will be skewed downward because of susceptibility gradients, it will cross the origin faster and the echo time will be shorter than originally planned. The same derivation works if the susceptibility gradient is negative which is the opposite direction of the gradients without susceptibility. The starting point of $k$-space trajectory will be moved to upward, the trajectory will be skewed upward, and the echo time will be longer. 


\subsection{Effect of Echo Time Shift on BOLD Signal}

If all tissue had the same susceptibility, then there will be no susceptibility-induced magnetic field gradients and no wrong echo time. However, in real cases, there are gradients in the magnetic field due to magnetic susceptibility variations near air/tissue interfaces. The susceptibility gradients will shift and skew the $k$-space trajectory. The echo time is measured at the origin (or at the point which has the minimum distance from the origin), but the echo time can be different than expected if the $k$-space trajectory is shifted and skewed. BOLD FMRI is acquired with an echo time that is chosen to optimize the contrast for the functional signal. If the echo time shifts, then this will affect the BOLD signal intensity.

We can quantify the BOLD signal through Percent signal change, PSC. Without susceptibility gradients, PSC is defined as

$$
\operatorname{PSC}\left(T E_{\text {nom }}\right)=100 * \frac{S_{\text {active }}-S_{\text {rest }}}{S_{\text {rest }}}=100 * \frac{S_{0}\left(\mathrm{e}^{-\frac{T E_{\text {nom }}}{T_{2, \text { cctive }}^{*}}}-\mathrm{e}^{-\frac{T E_{\text {nom }}}{T_{2, \text { rest }}^{*}}}\right)}{S_{0} \mathrm{e}^{-\frac{T E_{\text {nom }}}{T_{2, \text { rest }}^{*}}}}
$$

where $S_{\text {active }}$ is the FMRI signal received during the task when the tissue is active, $S_{\text {rest }}$ is the signal received during rest, $S_{0}$ is the voxel image intensity without $T_{2}^{*}$ relaxation, $T E_{\text {nom }}$ is the nominal echo time, $T_{2, \text { rest }}^{*}$ is the $T_{2}^{*}$ relaxation time constant during resting state, and $T_{2 \text {,active }}^{*}$ is the $T_{2}^{*}$ relaxation time constant during active state. In the past work, $T E_{\text {nom }}=30.0 \mathrm{~ms}$, $T_{2, \text { active }}^{*}=49.6 \mathrm{~ms}$ and $T_{2, \text { rest }}^{*}=48.9 \mathrm{~ms}$ [5].

If susceptibility gradients are added, then $\mathrm{k}$ space will be shifted and echo time will be changed as well [1]. PSC with susceptibility gradients can be defined in the similar way:

$$
\operatorname{PSC}\left(T E_{\text {eff }}\right)=100 * \frac{S_{0}\left(\mathrm{e}^{-\frac{T E_{\text {eff }}}{T_{2, \text { cctive }}^{*}}}-\mathrm{e}^{-\frac{T E_{\text {eff }}}{T_{2, \text { rest }}}}\right)}{S_{0} \mathrm{e}^{-\frac{T E_{\text {eff }}}{T_{2, \text { rest }}}}}
$$

where $T E_{\text {eff }}$ is the effective TE which is the echo time after counting susceptibility gradients.

\subsection{Calibration Function}

The information in the measured field map predicts how the echo time will shift and how to estimate the BOLD signal. A calibration function can be formed to remove the expected variation in the BOLD signal due to susceptibility gradients.

The calibration function, or percent signal change ratio, is defined as

$$
\text { Calibration }\left(T E_{\text {eff }}\right)=\frac{\operatorname{PSC}\left(T E_{\text {eff }}\right)}{\operatorname{PSC}\left(T E_{\text {nom }}\right)}
$$


The study of how the function of our brain changes with age will enlighten us on how to preserve our mind late into life and will provide information on healthy age-related declines in function versus other pathological changes such as Alzheimer's Disease. Much research is being conducted to better understand relationships between FMRI signal increases and their direct relationship to improvement or decrements in behavioral performance across age [6] [7]. Cabeza pointed out that the most powerful studies of the cognitive neuroscience of aging use functional neuroimaging techniques such as functional magnetic resonance imaging (FMRI) [8]. Recent FMRI studies have shown evidence of significant age-related changes in brain function in areas involved with memory, executive control, attention, motor control, and others. Specifically, common findings in the literature demonstrate that aging is associated with changes in magnitude, extent of activation, or laterality. However, magnetic susceptibility can cause spatially-varying BOLD sensitivity in the brain [1] [9]. In order to have an accurate age-related study, we first need to remove these sensitivity bias artifacts. After finding percent signal change ratio and removing the expected variation in the BOLD signal due to susceptibility gradients, then future studies can examine if there are actual changes in brain function [10]. To determine the impact of these sensitivity changes, we can determine some areas in a brain that have significant changes in sensitivity with age and compare those to areas commonly found to have age-related functional changes. Frontal regions and cingulate are the regions that have been previously implicated in age-related functional changes and we expect that these regions, along with others, will demonstrate age-related sensitivity variations that may partially account for these previous findings.

\subsection{Material and Methods}

To compare between old and young, we used two different data sets from previous functional imaging studies. The older subject group included twenty-six old adults (59 - 78 years old, mean age 63.15, 19 females) [11] [12] [13]. The younger subject group consisted of thirty young adults (18 - 21 years old, mean age 18.8, 18 females) [14] [15]. Subject scans were performed using a Siemens (Erlangen, Germany) Allegra 3T MRI scanner. For the older group's magnetic field maps, the followings are the parameters that are used: $\mathrm{TE}=10.00,12.46 \mathrm{~ms}$, $\mathrm{TR}=700 \mathrm{~ms}$, field of view $=22 \mathrm{~cm}$, base resolution $=64$, phase resolution $=72$, 28 slices $4.00 \mathrm{~mm}$ thick, bandwidth $=260.42$. For the younger group's magnetic field maps were acquired with the same protocol except for the following changes: $\mathrm{TR}=1000 \mathrm{~ms}, 38$ slices $3.3 \mathrm{~mm}$ thick. We do not expect that these parameter differences would yield difference field map measures in our study.

For the units of the field maps we used Hz. Gradients of the field map were simply found by calculating the differences with the neighbor pixels in the field map and the units of the gradients were $\mathrm{Hz} / \mathrm{cm}$. Since the field map and T2-weighted scan are in subject-specific anatomical spaces, we need image registration in order to transform them into a standard space where all subjects can 
be compared. The FMRIB group, Functional Magnetic Resonance Imaging of the brain, provides image registration tool in the FSL software package. The standard image that was used was the template MNI (Montreal Neurological Institute) brain. Registrations of our data to this template were performed using FLIRT (FMRIB's Linear Image Registration Tool) in FSL (FMRIB Software Library). To find $T E_{\text {eff }}$, we need to find $K_{\text {eff }}$ which is the sum of $K_{\text {imaging }}$ and $K_{\text {susc }}$. After finding $T E_{\text {eff }}$, by the definition of $\operatorname{PSC}\left(T E_{\text {eff }}\right)$ and calibration $\left(T E_{\text {eff }}\right)$, a calibration function in each voxel can be calculated.

After finding $T E_{\text {eff }}$ and Calibration $\left(T E_{\text {eff }}\right)$ of both the young and older group data, we used two-tailed t-test to compare the two groups of data on a voxel-by-voxel basis. By using Harvard-Oxford Structural Atlas in FSL, we ran t-test for specific anatomical regions of interest (ROIs) in the brain.

\section{Results and Discussion}

After running $\mathrm{t}$-tests for each of the ROIs and comparing the $\mathrm{p}$-values, we were found several areas that have significant difference between old and young subjects. Table 1 shows those areas. And Figure 4 is a bar chart that shows the means, standard deviations for both groups in each ROIs, standard deviations for both groups in each ROIs and its $-\log _{10}$ ( $p$-values), and an image of brain with some significant regions that have a big difference between ages (the blue regions mean the area where the p-value is between $10^{-2}$ to $10^{-5}$ ).

Magnetic field variations can result in changes in sensitivity of functional imaging in different brain regions. This can be problematic if there is a net bias between two groups that are being compared for brain function. Net bias in sensitivity can appear as functional changes across age, or can introduce additional variance and mask actual differences between groups. We have shown that there

Table 1. Areas that have significant difference.

\begin{tabular}{|c|c|c|c|c|c|}
\hline ROI & $\begin{array}{c}\text { mean } \\
(\text { Kramer })\end{array}$ & $\begin{array}{l}\text { mean } \\
\text { (Miller) }\end{array}$ & $\begin{array}{c}\text { std } \\
(\text { Kramer})\end{array}$ & $\begin{array}{c}\text { std } \\
\text { (Miller) }\end{array}$ & $p$-value \\
\hline Insular Cortex & $9.99 \mathrm{E}-01$ & $1.01 \mathrm{E}+00$ & $1.23 \mathrm{E}-02$ & $2.04 \mathrm{E}-02$ & $4.83 \mathrm{E}-03$ \\
\hline $\begin{array}{c}\text { Inferior Frontal } \\
\text { Gyrus, pars triangularis }\end{array}$ & $9.73 \mathrm{E}-01$ & $1.00 \mathrm{E}+00$ & $2.58 \mathrm{E}-02$ & $3.75 \mathrm{E}-02$ & $8.69 \mathrm{E}-04$ \\
\hline $\begin{array}{c}\text { Superior Temporal } \\
\text { Gyrus, posterior division }\end{array}$ & $1.00 \mathrm{E}+00$ & $1.04 \mathrm{E}+00$ & $1.72 \mathrm{E}-02$ & $2.39 \mathrm{E}-02$ & $6.41 \mathrm{E}-07$ \\
\hline $\begin{array}{c}\text { Middle Temporal } \\
\text { Gyrus, anterior division }\end{array}$ & $8.68 \mathrm{E}-01$ & $9.31 \mathrm{E}-01$ & $5.17 \mathrm{E}-02$ & $4.91 \mathrm{E}-02$ & $1.99 \mathrm{E}-05$ \\
\hline $\begin{array}{c}\text { Parahippocampal } \\
\text { Gyrus, anterior division }\end{array}$ & $9.47 \mathrm{E}-01$ & $1.02 \mathrm{E}+00$ & $5.78 \mathrm{E}-02$ & $7.37 \mathrm{E}-02$ & $1.89 \mathrm{E}-04$ \\
\hline Occipital Fusiform Gyrus & $1.01 \mathrm{E}+00$ & $9.80 \mathrm{E}-01$ & $2.20 \mathrm{E}-02$ & $2.16 \mathrm{E}-02$ & $6.78 \mathrm{E}-05$ \\
\hline Planum Polare & $9.52 \mathrm{E}-01$ & $9.86 \mathrm{E}-01$ & $1.76 \mathrm{E}-02$ & $2.51 \mathrm{E}-02$ & $3.81 \mathrm{E}-07$ \\
\hline $\begin{array}{c}\text { Heschl's Gyrus } \\
\text { (includes } \mathrm{H} 1 \text { and } \mathrm{H} 2 \text { ) }\end{array}$ & $9.94 \mathrm{E}-01$ & $1.01 \mathrm{E}+00$ & $6.63 \mathrm{E}-03$ & $1.15 \mathrm{E}-02$ & $1.90 \mathrm{E}-08$ \\
\hline Planum Temporale & $1.01 \mathrm{E}+00$ & $1.03 \mathrm{E}+00$ & $9.27 \mathrm{E}-03$ & $1.61 \mathrm{E}-02$ & $1.37 \mathrm{E}-05$ \\
\hline
\end{tabular}




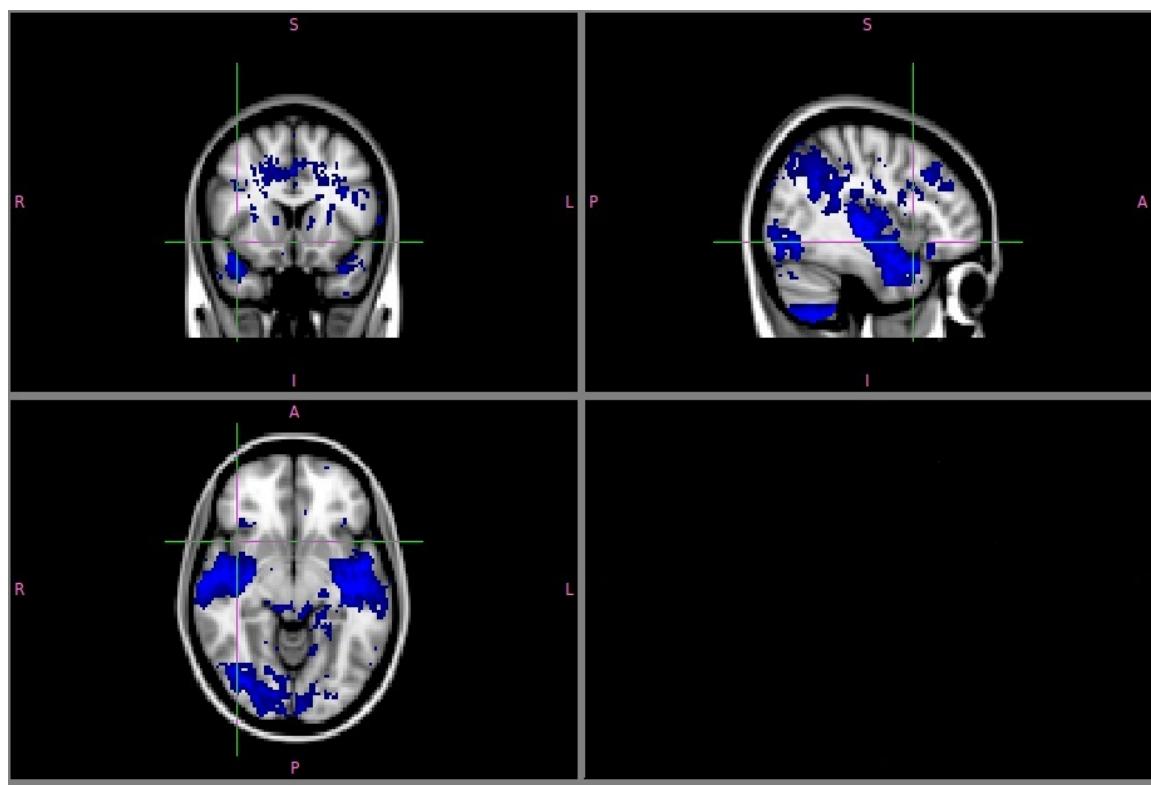

Figure 4. Areas that have significant difference between old and young subject of $-\log _{10}(p$-values $)$. This figure shows the means and standard deviation.

are net bias differences in old versus young adults in several regions that have been implicated in age-related changes in brain function. A thorough understanding of the relationship between the measurement technique and the interaction with other potential net changes between groups must be understood to ensure proper interpretation of functional differences. Calibration may help in eliminating the sensitivity bias between two groups, by measuring the individual specific magnetic field distributions and calculating the expected BOLD sensitivities [16].

There are several reasons why age-related changes in susceptibility-induced gradients may exist. One of the possible reasons is structural changes that accompany aging. Previous studies have used voxel-based morphometry (VBM) to assess changes in the overall structure of the brain accompanying age [12]. As tissue loss of the brain occurs with age, the magnetic field can be different between young and old people because the air/tissue interfaces and their relationship to other structures in the brain will be changed. In addition to anatomical differences that may affect air/tissue interfaces, age-related results from susceptibility weighted imaging shows that iron distributions in the brain can change with age. As people get older, iron concentrations in some areas change and it can cause a change in the magnetic field distribution. Another reason that can result in changes in the distribution of magnetic field is the subjects' head orientation. Truong et al. mentioned that the tilted head can significantly reduce the magnetic field gradients or significantly increase depending on which region of the brain is being examined [2], based on how the angle between the air/tissue interface and $\mathrm{B} 0$ will be changed. We measured the tilted angle of the subjects from our registration to the template image to determine by how much the images are rotated. The rotation angles for old subjects are $0.088 \pm 0.067$ radian 
and the rotation angles for young subjects are $0.057 \pm 0.063$ radian. The corresponding $p$-value is 0.087 which means that the rotation angles between old and young subjects are showing a trend towards being different, approaching significance. Generally, people become stooped as they are becoming older and this fact might cause this rotation angle difference.

Define abbreviations and acronyms the first time they are used in the text, even after they have been defined in the abstract. Abbreviations such as IEEE, SI, MKS, CGS, sc, dc, and rms do not have to be defined. Do not use abbreviations in the title or heads unless they are unavoidable.

\section{Conclusion}

Susceptibility gradient can shift and skew $k$-space trajectories and it leads to echo time shift and BOLD sensitivity change. Correct the artifacts by percent signal change (PSC) and calibration function, it can be apply into many different areas such as age-related studies. Accounting for subject-specific changes in BOLD sensitivity will enable careful studies to be performed to determine how the brain changes with age and how we can slow declines in function with age. This paper analyzed the performance of correcting the variations of BOLD Signal due to Susceptibility Gradients with real data set and identified the computational issues that need to be improved.

\section{Conflicts of Interest}

The authors declare no conflicts of interest regarding the publication of this paper.

\section{References}

[1] Deichmann, R., Josephs, O., Hutton, C., Corfield, D.R. and Turner, R. (2002) Compensation of Susceptibility-Induced BOLD Sensitivity Losses in Echo-Planar FMRI Imaging. NeuroImage, 15, 120-135. https://doi.org/10.1006/nimg.2001.0985

[2] Truong, T., Clymer, B.D., Chakeres, D.W. and Schmalbrock, P. (2002) Three-Dimensional Numerical Simulations of Susceptibility-Induced Magnetic Field Inhomogeneities in the Human Head. Magnetic Resonance Imaging, 20, 759-770. https://doi.org/10.1016/S0730-725X(02)00601-X

[3] Reber, P.J., Wong, E.C., Buxton, R.B. and Frank, L.R. (1998) Correction of Off Resonance-Related Distortion in Echo-Planar Imaging Using EPI-Based Field Maps. Magnetic Resonance in Medicine, 39, 328-330. https://doi.org/10.1002/mrm.1910390223

[4] Sutton, B.P., Noll, D.C. and Fessler, J.A. (2003) Fast, Iterative Image Reconstruction for MRI in the Presence of Field Inhomogeneities. IEEE Transactions on Medical Imaging, 22, 178-188. https://doi.org/10.1109/TMI.2002.808360

[5] Fera, F., Yongbi, M.N., van Gelderen, P., Frank, J.A., Mattay, V.S. and Duyn, J.H. (2004) EPI-BOLD FMRI of Human Motor Cortex at $1.5 \mathrm{~T}$ and 3.0 T: Sensitivity Dependence on Echo Time and Acquisition Bandwidth. Journal of Magnetic Resonance Imaging, 19, 19-26. https://doi.org/10.1002/jmri.10440

[6] Woodard, J. and Sugarman, M. (2011) Functional Magnetic Resonance Imaging in 
Aging and Dementia: Detection of Age-Related Cognitive Changes and Prediction of Cognitive Decline. In: Pardon, M.C. and Bondi, M., Eds., Behavioral Neurobiology of Aging. Current Topics in Behavioral Neurosciences, Springer, Berlin, Heidelberg, 113-136. https://doi.org/10.1007/7854_2011_159

[7] Reuter-Lorenz, P.A. and Park, D.C. (2010) Human Neuroscience and the Aging Mind: A New Look at Old Problems. The Journals of Gerontology Series B: Psychological Sciences and Social Sciences, 65B, 405-415.

https://doi.org/10.1093/geronb/gbq035

[8] Cabeza, R. (2001) Cognitive Neuroscience of Aging: Contributions of Functional Neuroimaging. Scandinavian Journal of Psychology, 42, 277-286. https://doi.org/10.1111/1467-9450.00237

[9] Gorno-Tempini, M.L., Hutton, C., Josephs, O., Deichmann, R., Price, C. and Turner, R. (2002) Echo Time Dependence of BOLD Contrast and Susceptibility Artifacts. Neuroimage, 15, 136-142. https://doi.org/10.1006/nimg.2001.0967

[10] Brian, Z. and Rabia, D. (2017) An Efficient Multistage Algorithm for Full Calibration of the Hemodynamic Model from BOLD Signal Responses. International Journal for Numerical Methods in Biomedical Engineering, 33, 2875-2879. https://doi.org/10.1002/cnm.2875

[11] Erickson, K.I., Voss, M.W., Prakash, R.S., Basak, C., Szabo, A., Chaddock, L., Kim, J.S., Heo, S., Alves, H., White, S.M., Wojcicki, T.R., Mailey, E., Vieira, V.J., Martin, S.A., Pence, B.D., Woods, J.A., McAuley, E. and Kramer, A.F. (2011) Exercise Training Increases Size of Hippocampus and Improves Memory. Proceedings of the National Academy of Sciences of the United States of America, 108, 3017-3022. https://doi.org/10.1073/pnas.1015950108

[12] Voss, M.W., Prakash, R.S., Erickson, K.I., Boot, W.R., Basak, C., Neider, M.B., Simons, D.J., Fabiani, M., Gratton, G. and Kramer, A.F. (2012) Effects of Training Strategies Implemented in a Complex Videogame on Functional Connectivity of Attentional Networks. NeuroImage, 59, 138-148. https://doi.org/10.1016/j.neuroimage.2011.03.052

[13] Prakash, R.S. (2011) Cardiorespiratory Fitness and Attentional Control in the Aging Brain. Frontiers in Human Neuroscience, 4, 229. https://doi.org/10.3389/fnhum.2010.00229

[14] Warren, S.L. (2010) Effects of Adult Attachment and Emotional Distractors on Brain Mechanisms of Cognitive Control. Psychological Science, 21, 1818-1822. https://doi.org/10.1177/0956797610388809

[15] Spielberg, J.M., Miller, G.A., Engels, A.S., Herrington, J.D., Sutton, B.P., Banich, M.T. and Heller, W. (2011) Trait Approach and Avoidance Motivation Lateralized Neural Activity Associated with Executive Function. NeuroImage, 54, 661-670. https://doi.org/10.1016/j.neuroimage.2010.08.037

[16] Takashi, M., Torikai, H., Shimokawa, T. and Leibnitz, K. (2014) A Nonlinear Model of FMRI BOLD Signal Including the Trend Component. Proceedings of the International Joint Conference on Neural Networks, Beijing, 6-11 July 2014, 2579-2586. https://doi.org/10.1109/IJCNN.2014.6889646 